Supplement of Geosci. Model Dev., 9, 2055-2076, 2016

http://www.geosci-model-dev.net/9/2055/2016/

doi:10.5194/gmd-9-2055-2016-supplement

(c) Author(s) 2016. CC Attribution 3.0 License.

(c) (1)

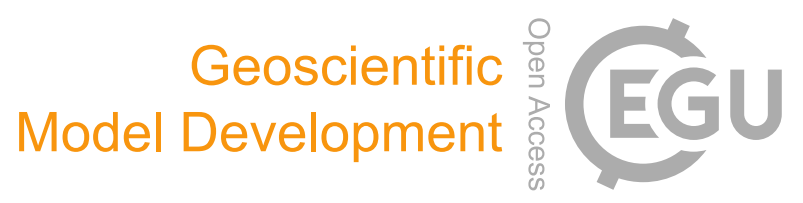

Supplement of

\title{
Randomly correcting model errors in the ARPEGE-Climate v6.1 component of CNRM-CM: applications for seasonal forecasts
}

\author{
Lauriane Batté and Michel Déqué \\ Correspondence to: Lauriane Batté (lauriane.batte@meteo.fr)
}

The copyright of individual parts of the supplement might differ from the CC-BY 3.0 licence. 
The figures in this document provide additional details on the stochastic dynamics perturbations and the analysis of their impact on seasonal re-forecast skill in experiments REF, SMM, S5D discussed in the paper. 

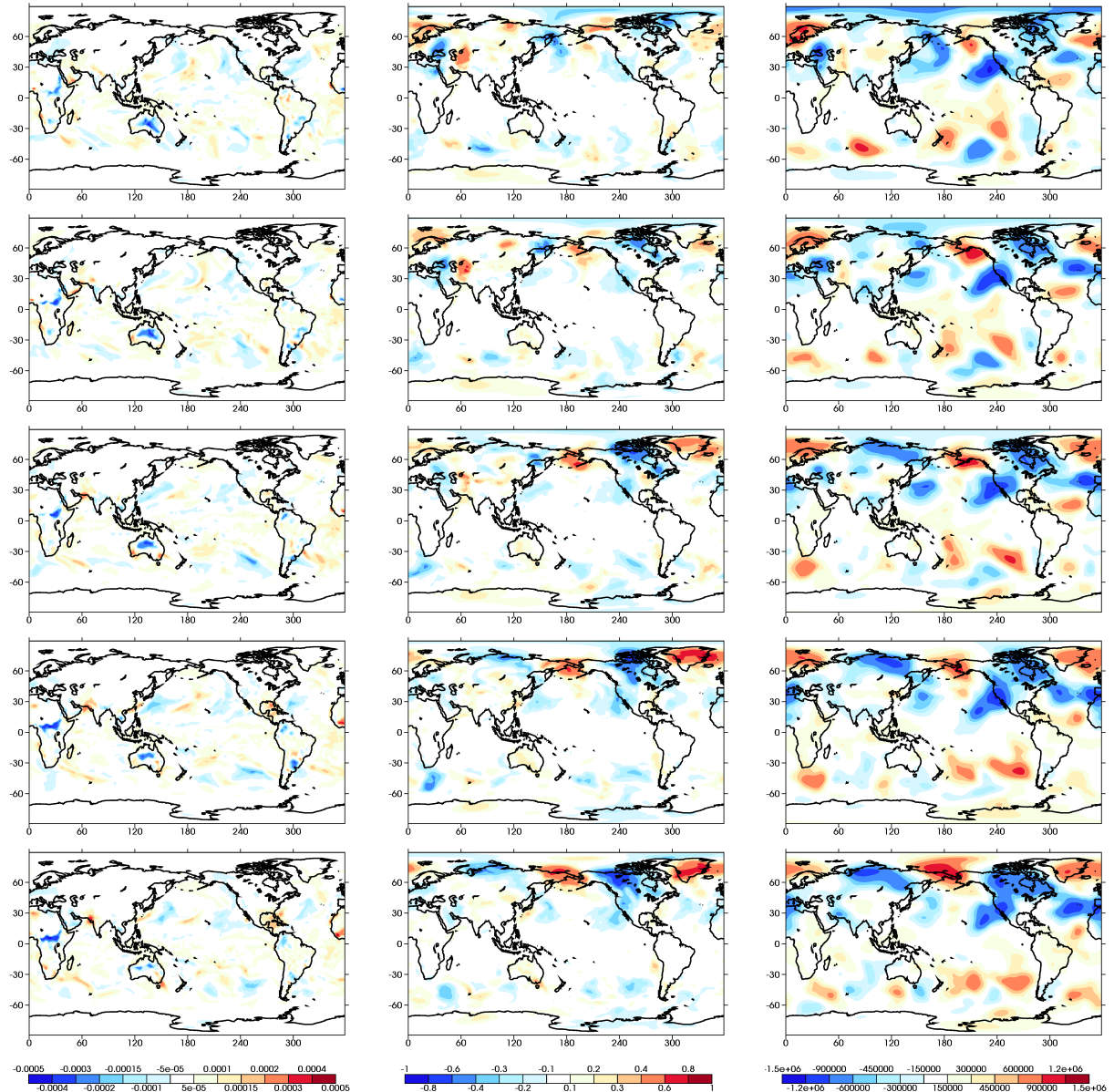

Figure S1: Example of corrections for humidity around $970 \mathrm{hPa}$, temperature at approximately $850 \mathrm{hPa}$ and streamfunction at around $500 \mathrm{hPa}$ (from left to right) for 5 consecutive days (from top to bottom) in February 1980 in the nudged re-forecast run. 

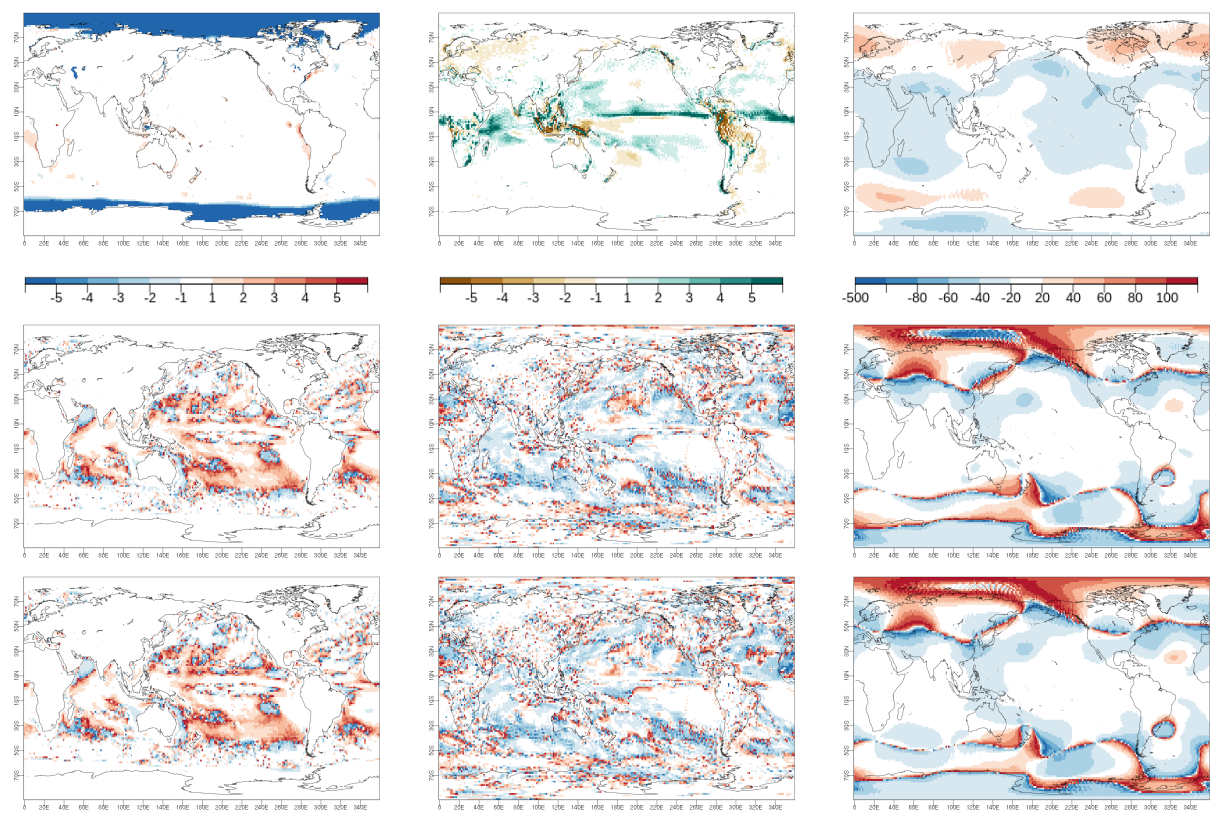

\begin{tabular}{lllllllllllll}
\hline & 0.1 & 0.25 & 0.5 & 0.75 & 0.9 & 1.1 & 1.25 & 1.5 & 2 & 5 & $1 \mathrm{e}+2 \mathrm{C}$
\end{tabular}

Figure S2: November bias (top row) for REF experiment SST, precipitation and Z500 (from left to right); corresponding relative absolute bias in experiments SMM and S5D (second and bottom rows, respectively). Bias is computed with respect to ERA-Interim for SST and GPCP for precipitation. Areas in blue indicate where bias is lower with respect to REF, whereas areas in shades of red show where bias is increased, regardless of the sign of the bias. 

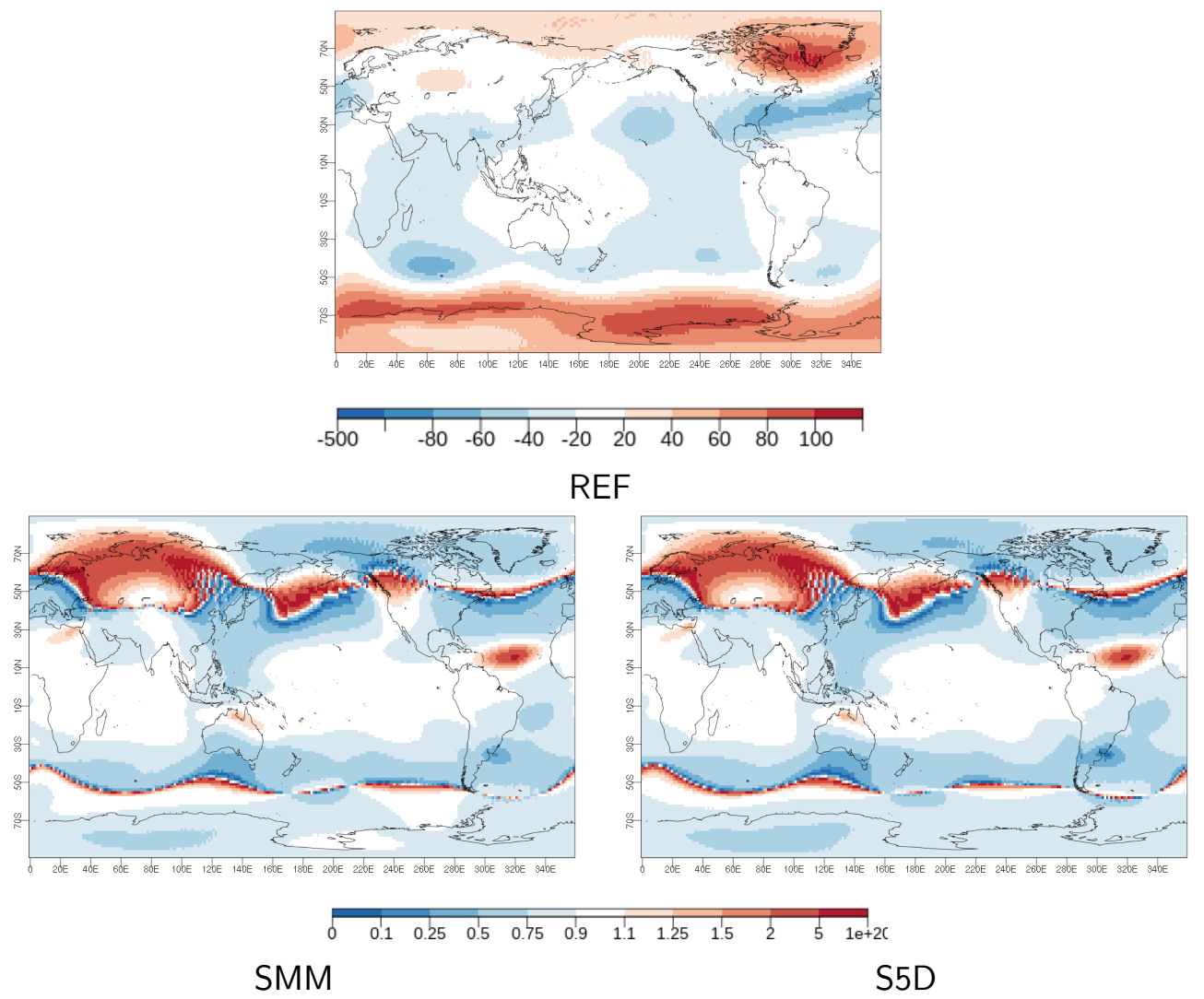

Figure S3: REF bias (top) for DJF $500 \mathrm{hPa}$ geopotential height over the globe with respect to ERA-Interim data. SMM (bottom left) and S5D (bottom right) relative absolute bias with respect to REF. 

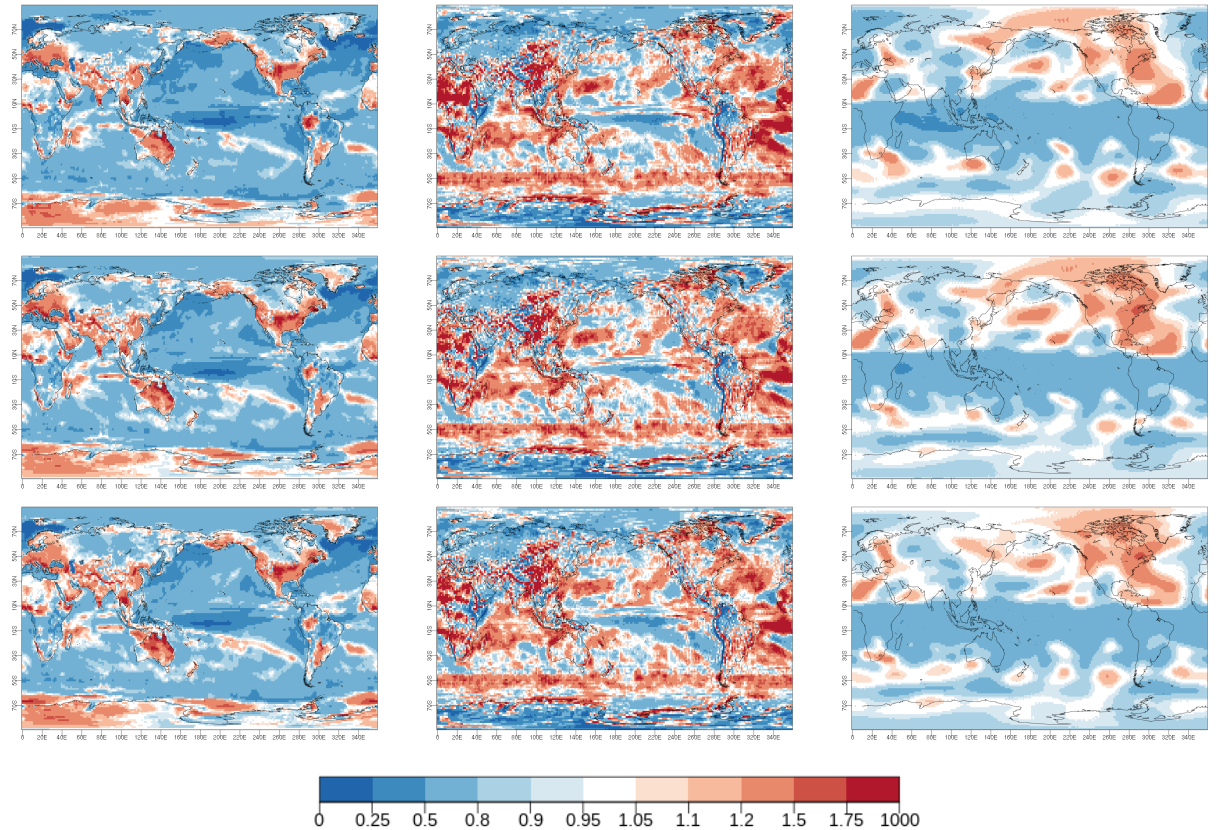

Figure S4: DJF spread-skill ratio for REF, SMM and S5D experiments (top to bottom rows, respectively) near-surface air temperature, precipitation and Z500 (from left to right). Spread is computed as the standard deviation around the ensemble mean, and skill is the ensemble mean root mean square error (RMSE). Areas in blue indicate where spread is lower than RMSE, whereas areas in shades of red show where spread is greater than RMSE. 


\section{SST > 2nd tercile}
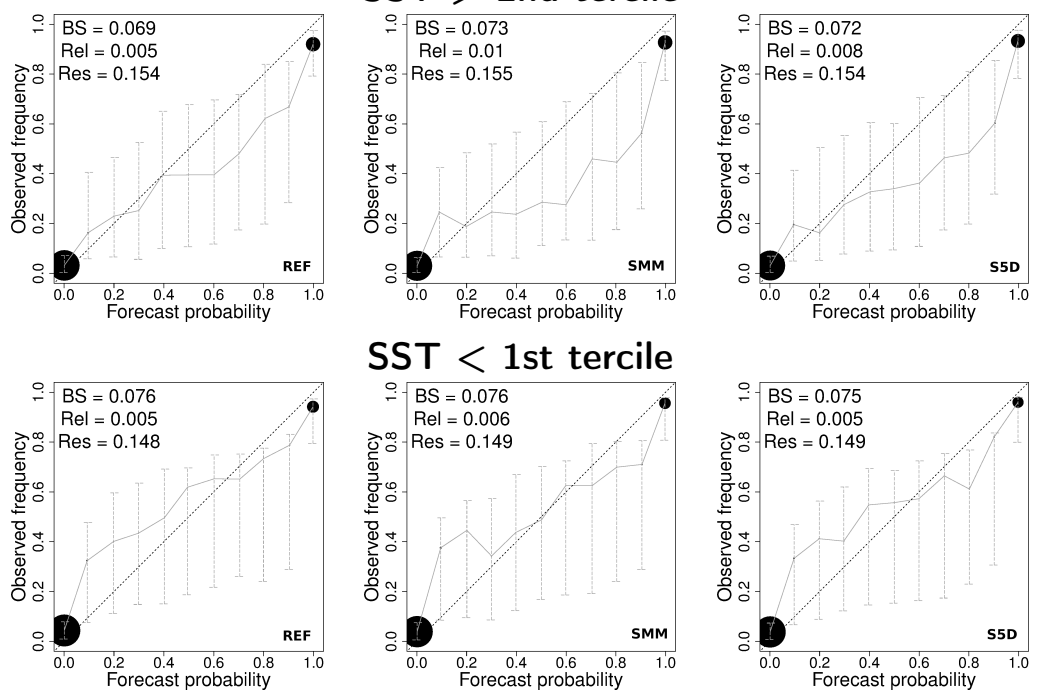

Figure S5: DJF Niño 3.4 reliability diagrams for SST exceeding the second tercile (top row) and remaining below the first tercile of reference climatology (bottom row), for experiments REF, SMM and S5D (from left to right). The Brier Score and reliability (Rel) and resolution (Res) decomposition is shown at the top left corner of each diagram. Dashed lines show the confidence intervals estimated by a bootstrapping method over the re-forecast period. The diameter of the circles is proportional to the size of the population bin for the different forecast probabilities. 


\section{TAS $>$ 2nd tercile}
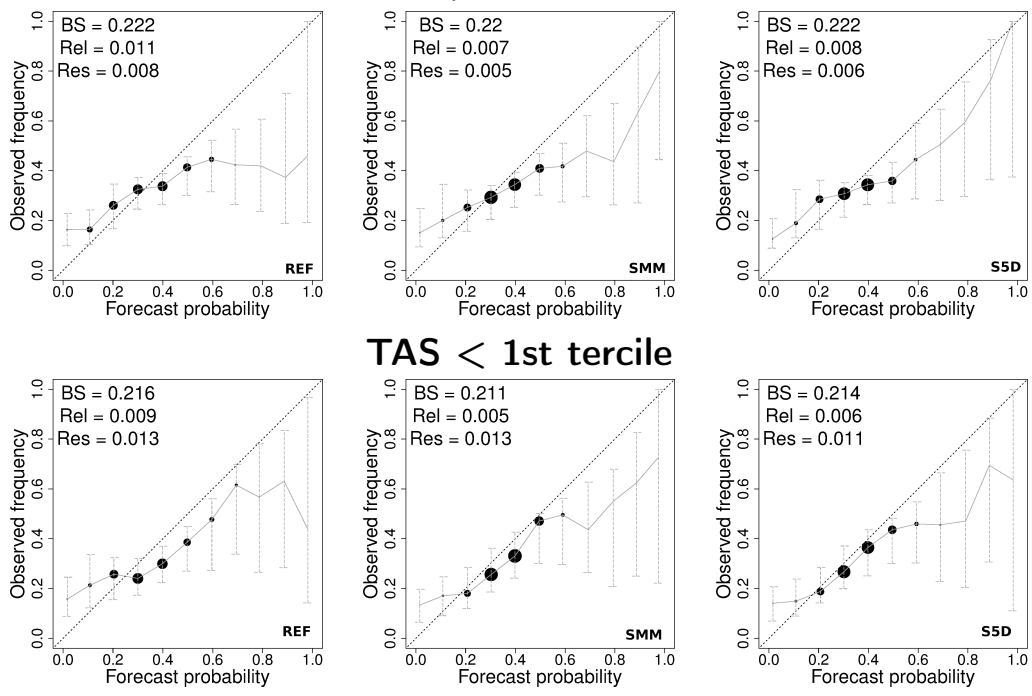

Figure S6: Same as figure S5 but for near-surface temperature over land in Europe verified against CRU data.
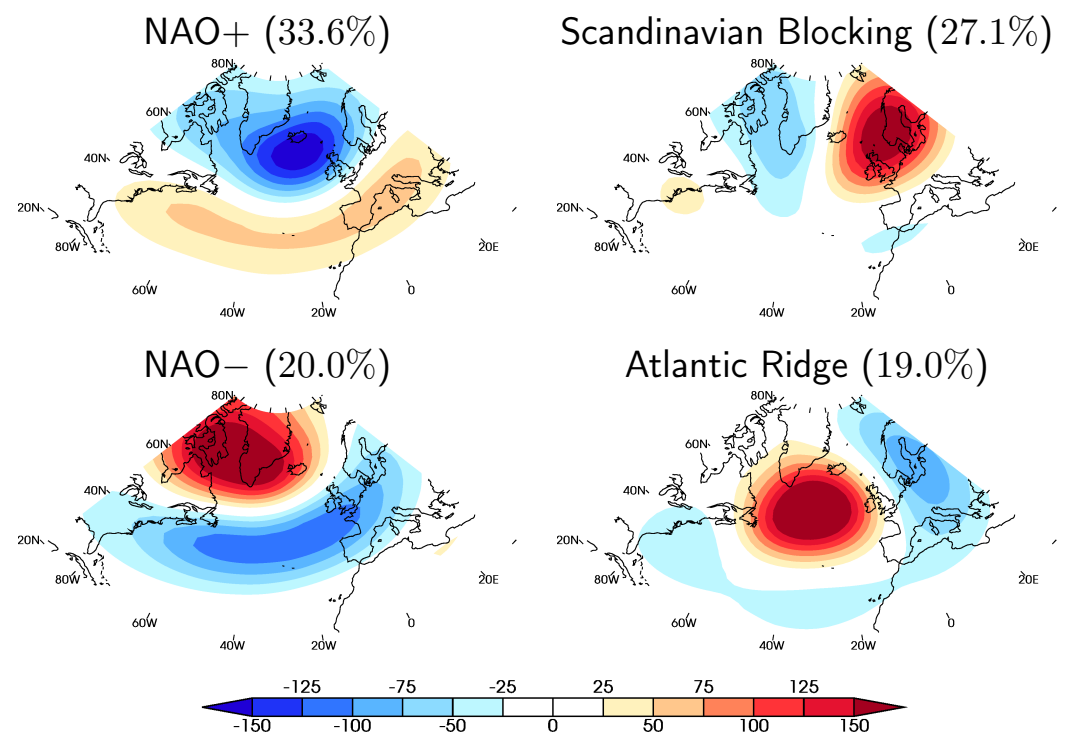

Figure S7: $500 \mathrm{hPa}$ geopotential height anomalies for the four North Atlantic weather regimes computed with ERA-Interim reanalysis data for DJF 1979-2012 

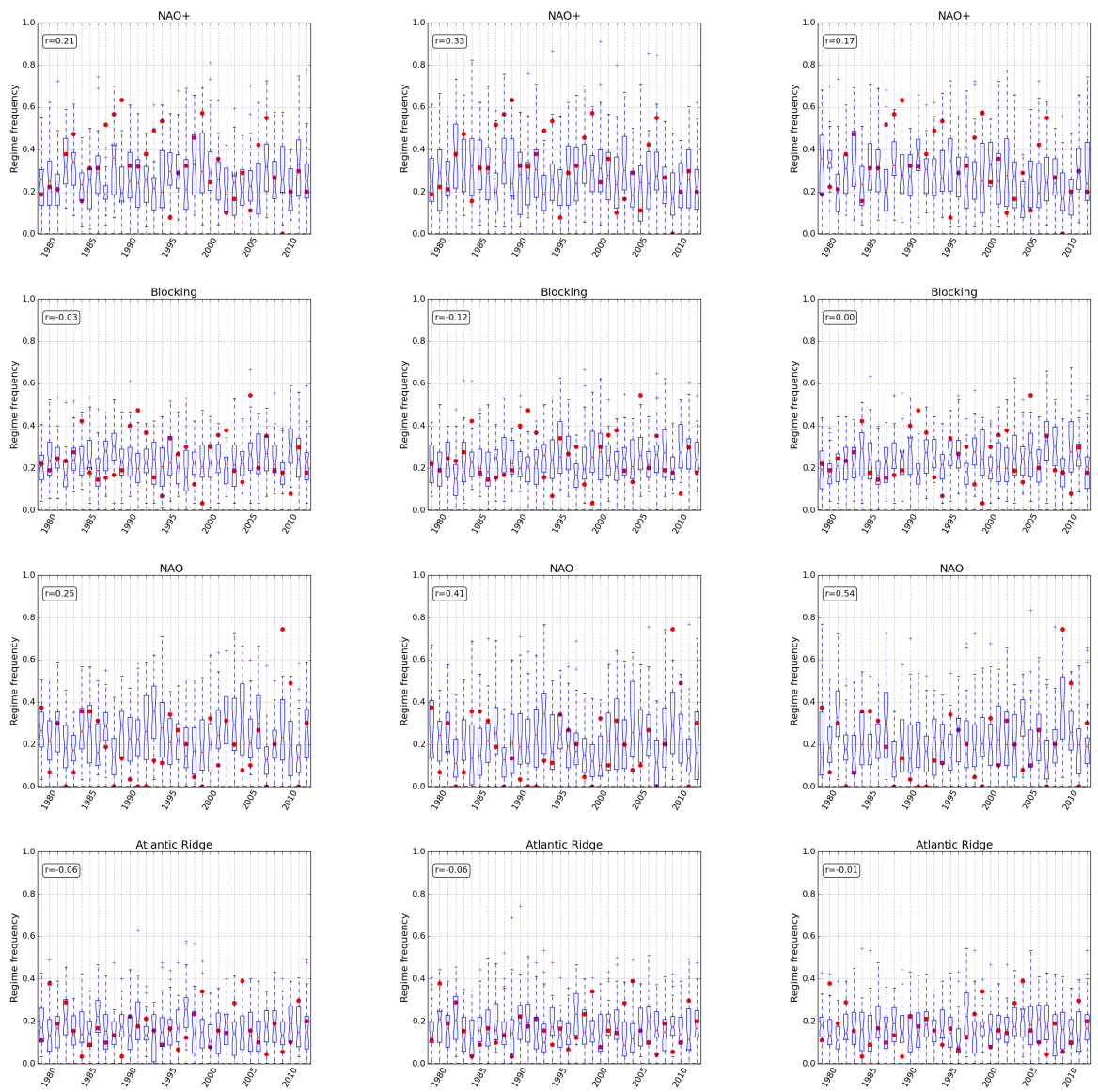

Figure S8: Boxplots for re-forecast ensemble DJF frequencies of each of the four North Atlantic weather regimes over the 1979-2012 time period in experiments REF (left) SMM (center) and S5D (right), and corresponding ERA-Interim frequency (red dots). Boxes show the interquartile range of the 30 ensemble members, while whiskers extend to a maximum of $\pm \sigma$. The correlation between ensemble mean and ERA-Interim frequencies is shown in the top left corner of each plot. 